KANSAS JOURNAL of MEDICINE

\section{Patterns of Injuries in Drowning Patients - Do These Patients Need a Trauma Team?}

Eric S. Hunn, M.D. , , Stephen D. Helmer, Ph.D., ${ }^{1,2}$ Jared Reyes, Ph.D. ${ }^{1}$, James M. Haan, M.D. ${ }^{1,3}$

${ }^{1}$ University of Kansas School of Medicine-Wichita, Department of Surgery, Wichita, KS

${ }^{2}$ Ascension Via Christi Saint Francis, Department of Medical Education, Wichita, KS

Received August 5, 2019; Accepted for publication May 5, 2020; Published online July 10, 2020

\section{ABSTRACT}

Introduction. Drowning is a major public health hazard worldwide, but associated traumatic injuries are rare. This study examined injuries and interventions performed on this population to assess the need for the trauma team activation.

Methods. A 12-year retrospective review was conducted on all fatal and non-fatal drowning patients who underwent a trauma work-up. Data collection included demographics, injury characteristics, interventions, and outcomes.

Results. Forty-three patients met inclusion criteria. Median patient age was six years (interquartile range 2 - 20) with $27.9 \%$ of patients under the age of 2 years. Most patients were white (62.8\%) and male (69.8\%), with median GCS score of 3 (60.5\% had initial GCS $=3$ with $25.6 \%$ with GCS $=15$ ). Only two patients suffered traumatic injuries. Only two patients required operations, neither of which suffered traumatic injury. Eleven patients suffered anoxic brain injury (25.6\%). Overall mortality was $48.8 \%(\mathrm{n}=21)$.

Conclusion. Patients who present with drowning and no traumatic mechanism have a very low rate of traumatic injuries. Work-up and treatment would be appropriate for emergency physicians without the need for a trauma activation. Kans J Med 2020;13:165-178

\section{INTRODUCTION}

Drowning is a major public health issue, annually claiming the lives of approximately 372,000 people worldwide. ' The World Health Organization defines drowning as, "The process of experiencing respiratory impairment from submersion/immersion in liquid.” Non-fatal drowning occurs when a patient is rescued and the process of drowning is interrupted. Fatal drowning occurs when a patient dies at any time after drowning. ${ }^{2}$ The pathophysiology of drowning is well-known by the medical community and described by multiple sources. ${ }^{2-4}$ In general, drowning starts as an isolated pulmonary process that leads to cardiac arrest within minutes without intervention. Water aspiration disrupts the normal surfactant present in the lungs, increases pulmonary compliance, and leads to hypoxia. ${ }^{2}$ Hypoxia then leads to bradycardia and cardiac arrest shortly thereafter.

Traumatic injuries rarely are identified in drowning patients. For example, Chotai and colleagues ${ }^{5}$ performed a retrospective study of 363 pediatric patients, only seven (1.9\%) of whom had traumatic soft tissue injuries. Furthermore, mortality was not affected by activating the trauma team in their study and only $2.2 \%$ were admitted to the pediatric trauma service. One concern with drowning patients is the possibility of cervical injuries from diving as a mechanism. ${ }^{6}$ According to Kewalramani and colleagues, ${ }^{6} 18 \%$ of their 126 spinal injuries were caused by diving accidents. Despite this concern, cervical injuries are rare when examining drowning patients as a whole. ${ }^{7,8}$ For example, Watson and colleagues ${ }^{7}$ found a very low incidence rate of cervical fractures when evaluating drowning patients in a large cohort study. They found the cervical injury incidence rate to be 11 of $2,244(0.5 \%)$ patients. Similarly, Hwang and colleagues ${ }^{8}$ performed a retrospective chart review of 143 pediatric drowning patients and they found a $4.9 \%$ incidence of traumatic injuries, all of which were cervical injuries. Hwang concluded that the prevalence of injury in drowning and near drowning is low and specialized trauma evaluations may not be necessary.

Despite these findings, drowning patients continue to trigger a trauma activation at many institutions across the nation including our level I trauma center. Trauma activations have a significant cost associated with them. ${ }^{9}$ The average trauma activation costs around $\$ 10,000$. Trauma activations require a large variety of hospital personnel to be available upon arrival of the patient to expedite care in an emergent fashion. These personnel include laboratory, radiology, respiratory, nursing, anesthesia, and a trauma surgeon.

Some studies supported management of drowning patients in the emergency department with a low percentage of patients needing admission to the hospital. ${ }^{10,11}$ For example, Noonan and colleagues ${ }^{10}$ performed a retrospective chart review of drowning patients and concluded that routine hospital admission is unnecessary after finding that the majority of symptomatic drowning patients (57\%) are asymptomatic after eight hours of observation in the emergency department. Similarly, $70.9 \%$ of Guzel and colleagues ${ }^{11}$ drowning patients were managed in the emergency department.

The purpose of this study was to evaluate the prevalence and patterns of traumatic injuries in drowning patients. With these data, we can help to determine if drowning patients necessitate a trauma activation, workup, and its associated cost. If these patients are found to have low rates of injuries and can be evaluated safely and treated without a trauma activation, then the cost of their hospital stay likely is to be significantly lower with likely a shorter length of stay.

\section{METHODS}

A retrospective chart review was conducted of all fatal and nonfatal drowning patients $(n=43)$ who presented as Level 1 or Level 2 traumas to an American College of Surgeons verified Level I trauma center from 2005 through 2017 and underwent a trauma work-up upon presentation. Patients were triaged by emergency personnel in the pre-hospital setting into level I or level II traumas or the emergency department for care upon arrival. The first responders triage patients depending upon the mechanism of injury, Glasgow Coma Scale score, and vital signs. All patients underwent assessment following the Advanced Trauma Life Support protocol upon arrival to the hospital. After the primary survey, if the patients were stable, a secondary survey was performed. Study patients were identified using the Trauma Registry and included all male and female sub- 
jects of any age. Data collection included demographics (age, gender, race), drowning location/mechanism (pool/other non-specific location, open water location, bathtub/suicide, diving accident), length of time submerged and interval to presentation, need for intubation, initial vital signs, presence of arrhythmias on presentation, injury details, imaging studies obtained, pulmonary complications, consultations, and outcomes (ICU admission and length of stay, mechanical ventilation and ventilator days, hospital length of stay, mortality, and disposition). Diving mechanism was included as an unspecified drowning location as it typically has been observed with associated injuries. Diving mechanism may have been in a pool or open water, but was only included in the diving mechanism category so that each category was mutually exclusive.

All data were taken directly from the electronic medical records (EMR). Race was reported as one of the following categories in the EMR: White or Caucasian, Black or African American, Asian, and Other. For our analysis, categories Asian and Other were combined to create one Other category.

This study was primarily descriptive in nature and as such data were summarized and reported as percentages or medians with interquartile ranges as appropriate for the respective data type. This study was approved for implementation by the institutional review board of Ascension Via Christi Hospitals Wichita, Inc.

\section{RESULTS}

A total of 43 patients met inclusion criteria (Table 1). The mean patient age was $15.9 \pm 20.0$ years, but the median age for patients was six years, with $27.9 \%$ of patients $(n=12)$ under the age of two. The majority of patients were white $(62.8 \%)$ males $(n=30,69.8 \%)$. Drowning locations included swimming pools (41.9\%), open water (23.3\%), bath tub (20.9\%), and diving at an unspecified location (14.0\%).

Table 1. Demographics and mechanism/location of injury of all drowning patients.

\begin{tabular}{|l|c|}
\hline Parameter & Number (\%) \\
\hline Number of subjects & $43(100 \%)$ \\
\hline Age (years)* & $6(2-20)$ \\
\hline Male & $30(69.8 \%)$ \\
\hline Race & \\
\hline White or Caucasian & $27(62.8 \%)$ \\
\hline Black or African American & $9(20.9 \%)$ \\
\hline Other & $7(16.3 \%)$ \\
\hline Drowning mechanism/location & \\
\hline Pool/other non-specific location & $18(41.9 \%)$ \\
\hline Open water location & $10(23.3 \%)$ \\
\hline Bathtub/suicide & $9(20.9 \%)$ \\
\hline Diving mechanism (unspecified location) & $6(14.0 \%)$ \\
\hline
\end{tabular}

*Presented as median (IQR)

The median time of submersion was five minutes and $55.8 \%$ of patients were intubated immediately upon or prior to arrival (Table 2). The median systolic blood pressure of study patients was $96 \mathrm{mmHg}$, however, approximately one-third of subjects arrived without palpable blood pressure $(n=16 ; 37.2 \%)$. Cardiac arrhythmias
KANSAS JOURNAL of MEDIC INE

INJURIES IN DROWNING PATIENTS

continued.

were common (54.8\%) and, of those, asystole was the most prevalent arrhythmia (87\%) with pulseless electrical activity being the next most common arrhythmia (13\%). Cardiopulmonary resuscitation was performed on $79.1 \%$ of the patients $(n=34)$. Most drowning patients were on one extreme of the Glasgow Coma Scale (GCS) or the other at arrival with a median GCS score of 3 (interquartile range of 3 to 15). For example, the majority of the patients had a GCS score of 3 (60.5\%), and a quarter (25.6\%) of the patients had a normal GCS score at 15 . Anoxic brain injury definitively was diagnosed in a quarter of the patients $(25.6 \%)$.

Table 2. Presenting characteristics and injury details of all drowning patients.

\begin{tabular}{|l|c|}
\hline Parameter & Value* \\
\hline Number of subjects & $43(100 \%)$ \\
\hline Time submerged (min.) & $5(2-10)$ \\
\hline Time to presentation (min.) & $51(37-78)$ \\
\hline Arrived intubated or intubated on arrival & $24(55.8 \%)$ \\
\hline Initial vital signs & \\
\hline Heart rate & $104(0-121)$ \\
\hline Respiratory rate & $17.5(0-30)$ \\
\hline Body temperature $\left({ }^{\circ} \mathrm{F}\right)$ & $96.4(95.0-97.6)$ \\
\hline Systolic blood pressure $(\mathrm{mmHg})$ & $96(0-123)$ \\
\hline Diastolic blood pressure $(\mathrm{mmHg})$ & $58(0-85)$ \\
\hline Arrhythmia present on arrival & $23 / 42(54.8 \%)$ \\
\hline Asystole & $20(87 \%)$ \\
\hline Pulseless electrical activity & $3(13 \%)$ \\
\hline Traumatic injuries & $2(4.7 \%)$ \\
\hline Clavicle fracture & $1(2.3 \%)$ \\
\hline Cervical injury & $1(2.3 \%)$ \\
\hline Anoxic brain injury & $11(25.6 \%)$ \\
\hline
\end{tabular}

*Values presented as number (\%) or median (IQR).

Traumatic injuries were rare with only two patients (4.7\%) suffering traumatic injuries, excluding those with skin abrasions/ lacerations and contusions. One of the two patients that did suffer a traumatic injury was a 54-year-old male who was found in a lake with suspected diving mechanism. He suffered a complex $\mathrm{Cl}$ fracture involving bilateral lamina and the anterior arch. He also sustained a C2 odontoid fracture as well as a vertebral artery dissection and a subarachnoid hemorrhage. This patient was diagnosed with brain death and underwent organ donation only a few days after arrival. The second patient suffering injury was an elderly gentleman who was on hospice for lung disease and heart failure when he fell into a bathtub and suffered a clavicle fracture. He quickly deteriorated and the family elected for comfort care shortly after his arrival to the hospital.

Only two of the patients (4.7\%) required operative intervention, none of which were emergent in nature or associated with traumatic injuries. One patient was found in a swimming pool and had 
KANSAS JOURNAL of MEDICINE

INJURIES IN DROWNING PATIENTS

\section{continued.}

cardiopulmonary resuscitation performed, regaining spontaneous circulation at the scene. He eventually was diagnosed with anoxic brain injury and had subsequent dysphagia requiring a surgical gastrostomy tube placement. The second patient requiring operative care was a 9-year-old male that also drowned in a pool and suffered from anoxic brain injury. He underwent a tracheostomy, gastrostomy, dental wiring, and tongue wound debridement after a prolonged hospital stay.

Study patients underwent a variety of imaging studies (Table 3). Nearly every patient received a chest x-ray (79.1\%) and focused assessment with sonography for trauma (FAST) exam (79.1\%). Computed tomography of the head also was common (41.9\%). Pulmonary complications were observed in $18.6 \%$ of the patients $(n=8)$. The most common pulmonary complication was pulmonary edema (11.6\%; $\mathrm{n}=5$ ), followed by pneumonia in two patients $(4.7 \%)$, and Acute Respiratory Distress Syndrome in one patient (2.3\%).

Table 3. Radiographic imaging studies performed for all drowning patients.

\begin{tabular}{|l|c|}
\hline Parameter & Number (\%) \\
\hline Number of subjects & $43(100 \%)$ \\
\hline Chest x-ray & $34(79.1 \%)$ \\
\hline Focused assessment with sonography for trauma & $34(79.1 \%)$ \\
\hline Computed tomography of head & $18(41.9 \%)$ \\
\hline Computed tomography of cervical spine & $7(16.3 \%)$ \\
\hline Kidney, ureter, and bladder (KUB) x-ray & $6(14.0 \%)$ \\
\hline Pelvic x-ray & $4(9.3 \%)$ \\
\hline Computed tomography of neck & $2(4.7 \%)$ \\
\hline Computed tomography of lumbar spine & $2(4.7 \%)$ \\
\hline Computed tomography of thoracic spine & $1(2.3 \%)$ \\
\hline Computed tomography angiography of neck & $1(2.3 \%)$ \\
\hline Cerebral angiogram & $1(2.3 \%)$ \\
\hline Cerebral scan & $1(2.3 \%)$ \\
\hline Head magnetic resonance imaging & $1(2.3 \%)$ \\
\hline
\end{tabular}

The median hospital length of stay was one day and $55.8 \%$ of patients were admitted to the ICU (Table 4). The overall mortality was high among drowning patients (48.8\%); four were pronounced dead on arrival, nine were pronounced dead in the emergency department or trauma bay, and eight died after admission. Six of eight patients that expired after admission were made comfort care. Consults were obtained in one-third of patients (34.9\%); however, care was never transferred to another service. While mortality was high in this population, 19 patients (44.2\%) were discharged home, with one additional patient each being discharged to rehabilitation, a mental health facility, and an acute care hospital (Table 5).
Table 4. Consultations and hospital outcomes for all drowning patients.

\begin{tabular}{|l|c|}
\hline Parameter & Number (\%) \\
\hline Number of subjects & $43(100 \%)$ \\
\hline Consulted non-trauma/surgical intensivist & $15(34.9 \%)$ \\
\hline Transferred care to non-trauma/surgical intensivist & $0(0 \%)$ \\
\hline Intensive Care Unit admission & $24(55.8 \%)$ \\
\hline Intensive Care Unit days* & $1(1,2)$ \\
\hline Mechanical ventilation & $13(30.2 \%)$ \\
\hline Ventilator days* & $2(1,3)$ \\
\hline Hospital length of stay* & $1(1,2)$ \\
\hline Comfort care & $6(14.0 \%)$ \\
\hline Mortality & $21(48.8 \%)$ \\
\hline Dead on arrival & $4(19.0 \%)$ \\
\hline Death in emergency department or trauma bay & $9(42.9 \%)$ \\
\hline Died after admission & $8(38.1 \%)$ \\
\hline
\end{tabular}

*Values presented as median (IQR)

Table 5. Discharge disposition for all drowning patients.

\begin{tabular}{|l|c|}
\hline Discharge Location & Number (\%) \\
\hline Number of subjects & $43(100 \%)$ \\
\hline Home & $19(44.2 \%)$ \\
\hline Rehabilitation center & $1(2.3 \%)$ \\
\hline Mental health facility & $1(2.3 \%)$ \\
\hline Acute care hospital & $1(2.3 \%)$ \\
\hline Dead on arrival & $4(9.3 \%)$ \\
\hline Death in emergency department or trauma bay & $9(20.9 \%)$ \\
\hline Death in hospital & $8(18.6 \%)$ \\
\hline
\end{tabular}

\section{DISCUSSION}

Prevalence of Associated Injuries. Drowning and near drowning patients have a very low rate of associated traumatic injuries. ${ }^{5,7,8}$ Only one patient (2.4\%) in our study suffered a cervical injury, which is similar to the findings of Hwang et al. ${ }^{8}$ who found that $4.9 \%$ of drowning patients had injuries, all of which were cervical in nature. In addition, out of Hwang's drowning population, all but one of the patients with a cervical injury had a witnessed diving mechanism. Similarly, Watson et al. ${ }^{7}$ found traumatic cervical injuries in only 11 (0.5\%) of 2,244 patients. All 11 of the injured patients had clinical signs of serious injury or a witnessed traumatic mechanism such as a dive, motor vehicle collision, or fall from a height. These are comparable to the only patient suffering a cervical injury having a suspected diving mechanism upon presentation in our study. This supported the evidence of witnessed diving or other significant traumatic mechanisms and subsequent drowning justifying a trauma activation based on mechanism alone. Chotai et al. ${ }^{5}$ also found minimal traumatic injuries in their study of 363 pediatric drowning and near drowning patients. They only found soft tissue injuries in seven patients (1.9\%).

Need for 'Trauma Team Activation. Chotai et al. ${ }^{5}$ found that only $2.2 \%$ of the 363 drowning and near drowning children were admitted to the pediatric trauma service due to the low incidence of surgical interventions at presentation. The majority of patients from our study died shortly upon arrival or had very brief hospital stays for 
observation and were discharged home on hospital day two. Even though $79 \%$ of the patients in our study received cardiopulmonary resuscitation upon presentation, these patients could have been managed by emergency personnel skilled in medical advanced cardiac life support. This would avoid the high cost associated with a trauma activation and significantly reduce the amount of hospital personnel on standby during an ongoing trauma activation. Due to the brief length of hospital stay observed by most surviving patients, any acute care service or hospitalist service could manage these patients appropriately and consult specialists on an as needed basis. These findings were comparable to Noonan and colleagues ${ }^{10}$ who concluded that routine hospital admission of all children who have had an immersion accident is unnecessary. They found that the majority (57\%) of symptomatic drowning patients are asymptomatic after eight hours of observation in the emergency department and by 18 hours of observation $72 \%$ were asymptomatic. Guzel et al. ${ }^{11}$ also observed that $70.9 \%$ of their drowning patients were followed in the emergency department. Thus, data from our study as well as that of others indicated that the majority of drowning patients do not necessitate a trauma activation and that most can be managed by an acute care service.

Need for Operative Intervention. Only two of the 43 patients in this study $(4.7 \%)$ required an operation and none of the operations performed were during the day of presentation, as a result of trauma, or emergent in nature. Both patients requiring operative intervention suffered anoxic brain injuries after being found in a pool. These results were similar to the findings of Chotai et al. ${ }^{5}$ where only two $(0.006 \%)$ of the patients received surgical intervention within 24 hours of presentation. These interventions included a bronchoscopy and extracorporeal membrane oxygenation. This evidence supported that a trauma surgeon and a trauma service are not needed to aid in the initial management of drowning patients due to the very low rate of traumatic injuries in this population and the low rate of operations needed in the acute setting.

Limitations. This study was limited by the low power and few numbers of drowning patients meeting inclusion criteria. Additional retrospective studies with more power are needed to verify these findings. The study was also retrospective in nature, with all the limitations inherent in that study design, and included all ages over a large timeframe (12 years). Future studies could evaluate outcomes of drowning patients that have been managed by emergency physicians or trauma teams and evaluate if there are statistical differences in mortality, hospital length of stay, injuries identified, ventilator days, and cost of admission.

\section{CONCLUSION}

Patients who presented with the sole mechanism of drowning or near drowning, and no witnessed or suspected traumatic mechanism, have a low rate of traumatic injuries. Work-up and treatment would be appropriate for emergency physicians without the need for a trauma activation. This would save patients the high cost of a trauma activation and allow the trauma services to direct their resources to patients with more severe injuries who may require the attention of a trauma surgeon.
KANSAS JOURNAL of MEDICINE

INJURIES IN DROWNING PATIENTS

continued.

\section{ACKNOWLEDGEMENT}

This project was presented at the 7lst Annual Meeting of the Southwestern Surgical Congress, April 14 - 17, 2019, Huntington Beach, California.

\section{REFERENCES}

1 World Health Organization. Global report on drowning: Preventing a leading killer. 2014. Available at: https://apps.who.int/iris/bitstream/handl e/10665/143893/9789241564786_eng.pdf;jsessionid=2135D7EE93FA8 292EC7CADCDBF49D6F0?sequence=l. Accessed May 8, 2019.

${ }^{2}$ Szpilman D, Orlowski JP, Bierens J. Drowning. In: Vincent J, Abraham E, Moore FA, Kochanek PM, Fink MP. (Eds.). Textbook of Critical Care. 7th Edition. Philadelphia: Elsevier, 2017, pp 493-499.el.

3 Giammona ST. Drowning: pathophysiology and management. Curr Probl Pediatr 1971; 1(7):1-33. PMID: 4950507.

4 Ibsen LM, Koch T. Submersion and asphyxial injury. Crit Care Med 2002; 30(11 Suppl):S402-408. PMID: 12528781.

${ }^{5}$ Chotai PN, Manning L, Eithun B, et al. Pediatric near-drowning events: Do they warrant trauma team activation? J Surg Res 2017; 212:246. PMID: 28666527

${ }^{6}$ Kewalramani LS, Taylor RG. Injuries to the cervical spine from diving accidents J Trauma 1975; 15(2):130-142. PMID: 1113351.

7 Watson RS, Cummings P, Quan L, Bratton S, Weiss NS. Cervical spine injuries among submersion victims. J Trauma 2001; 51(4):658-662. PMID: 11586155.

${ }^{8}$ Hwang V, Shofer FS, Durbin DR, Baren JM. Prevalence of traumatic injuries in drowning and near drowning in children and adolescents. Arch Pediatr Adolesc Med 2003; 157(1):50-53. PMID: 12517194.

9 Stein L, Zayas A. Florida trauma centers charge outrageous fees the moment you come through the door. Tampa Bay Times. March 8, 2014, Available at: www.tampabay.com/news/health/florida-trauma-centerscharge-outrageous-fees-the-moment-you-come-through/2169148/\#talkwrapper. Accessed April 23, 2020.

10 Noonan L, Howrey R, Ginsburg CM. Freshwater submersion injuries in children: A retrospective review of seventy-five hospitalized patients. Pediatrics 1996; 98 (3 Pt 1):368-371. PMID: 8784358.

${ }^{11}$ Güzel A, Duran L, Paksu S, et al. Drowning and near-drowning: Experience of a university hospital in the Black Sea region. Turk J Pediatr 2013; 55(6):620-627. PMID: 24577981.

Keywords: drowning, injuries and wounds, trauma 